Bio - grafía. Escritos sobre la Biología y su Enseñanza. ISSN 2027-1034

Edición Extraordinaria. p.p. 411 - 419

Memorias del IX Encuentro Nacional de Experiencias en Enseñanza de la Biología y la Educación Ambiental. IV Congreso Nacional de Investigación en Enseñanza de la Biología.

\title{
ENRIQUE BELTRÁN CASTILLO PIONERO DE LA RACIONALID AD CONSERVACIONISTA EN MÉXICO
}

\author{
Vicente Paz Ruiz ${ }^{1}$
}

\section{RESUMEN}

Se hace un trabajo de investigación documental con la finalidad de reconocer la obra de Enrique Beltrán en el ámbito de la formación de profesionales de la enseñanza de la Biología para educación secundaria en México. Para ello se revisaron fuentes primarias y secundarias, se organizaron por: política educativa, ecología como disciplina y aporte de Beltrán al campo conservacionista. En el primer caso se revisó la política desarrollista iniciando con el cardenismo, en el segundo se ve a la ecología desde un enfoque sistémico y homeostásico, el aporte de Beltrán se ordena por currículo y obra escrita. Se concluye que Beltrán Castillo aporta sillares fundamentales para promover el enfoque conservacionista como respuesta educativa a la barbarie ecocida desarrollista.

PALABRAS CLAVE: Formación docente, Escuela normal, Ecología, Conservacionismo, Educación ambiental

\section{ABSTRAC}

A documentary research work is done with the purpose of recognizing the work of Enrique Beltrán in the field of the training of professionals of the teaching of biology for secondary education in Mexico. For this, primary and secondary sources were reviewed, organized by: educational politic, ecology as a discipline and Beltrán's contribution to the conservationist field. In the first case the developmental politic was revised from the cardenismo, in the second one, the ecology is viewed from a systemic and homeostatic approach, and the contribution of Beltran is ordered by curriculum and written work. It is concluded that Beltrán Castillo provides fundamental ashlars to promote the conservationist approach as an educational response to eco barbarism.

KEYWORDS: Teacher training, Normal school, Ecology, Conservationism, Environmental education

\footnotetext{
1 Universidad Pedagógica Nacional de México, Unidad 094 Centro, ypaz@upn.mx.Formación de Profesores de Biología y Educación Ambiental
} 
Bio - grafía. Escritos sobre la Biología y su Enseñanza. ISSN 2027-1034

Edición Extraordinaria. p.p. 411 - 419

Memorias del IX Encuentro Nacional de Experiencias en Enseñanza de la Biología y la

Educación Ambiental. IV Congreso Nacional de Investigación en Enseñanza de la Biología.

\section{INTRODUCCIÓN}

El Dr. Enrique Beltrán es un personaje de gran relevancia en el ámbito de las ciencias biológicas en México, nació a principios del siglo XX y murió en la última parte del mismo siglo, su actividad como investigador, profesor, diseñador de programas educativos y político coadyuvaron a la profesionalización de la Biología y su institucionalización en México.

Beltrán fue alumno directo de Alfonso L. Herrera, promotor de la aceptación de la teoría de la evolución en América, precursor de los estudios de origen de la vida y materialista convencido, su influencia en las nuevas generaciones se dio por sus escritos como el primer libro de Biología que se publicó en México pero sobre todo como maestro de otros que habrían de seguir el linaje en transición de naturalista a biólogos. Beltrán aportó en Biología conocimiento nuevo sobre protozoarios pero su gran aporte es haber formado a las nuevas generaciones de enseñantes de la Biología con un enfoque conservacionista que poco se ha reconocido por los educadores ambientales.

Este texto tiene como motivación el poco conocimiento y reconocimiento que se tiene en el ámbito de la Educación ambiental y en la enseñanza de la Biología en secundaria en México de Enrique Beltrán, una figura central para el desarrollo de la racionalidad conservacionista en el país. Más aún no aparece en textos de secundaria e incluso en los de formación de profesionales de la educación para dicha disciplina, lo cual es motivo de un desenraizamiento de la cultura magisterial actual con sus fundadores.

Esto es un problema por ello el propósito del trabajo es dar a conocer los aportes de Enrique Beltrán en el ámbito de la Enseñanza de la Biología y de la racionalidad conservacionista, embrión de la Educación ambiental a las nuevas generaciones de profesionales de la educación ambiental y de profesores de Biología de secundaria.

\section{METODOLOGÍA}

Para desarrollar este trabajo se recurrió a una búsqueda documental tanto de fuentes primarias (fondo de Secretaría de Educación Pública y documentos de Beltrán) así como de secundarias especializadas. Para ordenar dicha información se formaron tres organizadores temáticos: político - histórico, ecológico, curricular. Con ello se obtuvieron; un reconocimiento de la política educativa de la época desarrollista en México, una idea de lo que es la ecología y el conservacionismo y una descripción de los planes de estudio de la Escuela Normal Superior de México de 1936 a 1970 donde se incluye la obra y contenido de la misma sobre ecología y conservación de Beltrán. Para analizarlo se empleó un entrecruce entre los organizadores mencionados tomando como eje la obra de Beltrán ubicándolos en su contexto histórico. 
Bio - grafía. Escritos sobre la Biología y su Enseñanza. ISSN 2027-1034

Edición Extraordinaria. p.p. 411 - 419

Memorias del IX Encuentro Nacional de Experiencias en Enseñanza de la Biología y la Educación Ambiental. IV Congreso Nacional de Investigación en Enseñanza de la Biología.

\section{RESULTADOS}

Política educativa en el desarrollismo en México

En México durante la escuela Socialista del presidente Cárdenas (1934 - 1940), se promovió la idea de que la naturaleza fuera una fuente de recursos para el bienestar colectivo por ello su racionalidad fue ilustrada, con un fin benefactor para la mayoría, su tesis era:

Unificar el pensamiento de nuestra colectividad encauzando las acciones de las nuevas generaciones hacia la organización de un régimen en que la igualdad sea consecuencia de una equitativa distribución económica (...) la implantación de la escuela socialista significa un paso más hacia la redención material y espiritual de las clases asalariadas dentro de un orden de efectiva justicia social (...) poniendo la escuela al alcance de las clases laborantes. La educación estará encauzada preferentemente hacia las clases campesinas y obreras, vinculada con los problemas del medio las necesidades y aspiraciones de las agrupaciones proletarias y acorde con los progresos de la técnica para la socialización de la riqueza (...) la educación normal modifica su plan de estudios a uno de pedagogía dialéctica globalizando áreas evitando dispersión por asignaturas. Los complejos son: de la naturaleza, de la sociedad del trabajo (SEP, 1935, p.13).

La tesis socialista modificaba la educación y unificaba un área la del complejo de la naturaleza, relacionándolo con el trabajo y la sociedad, se buscaba que el conocimiento científico no se impartiera como un insumo de eruditos, o simple información cultural, se buscaba que estuviesen al servicio del colectivo por eso las relaciones trabajo, sociedad naturaleza, pues el conocimiento sobre la naturaleza era el centro de la actividad humana (SEP, 1937).

El programa de la Escuela Normal Superior de México, formadora de docentes para biología en secundaria buscaba que se conociera la naturaleza para dar al alumno una visión, lo más clara posible del medio que lo rodea y del que él mismo forma parte (una mirada ecologista), esto se expresa claramente en su segundo objetivo:

Segundo objetivo, será proporcionar el conocimiento de la naturaleza, la indicación concreta para el dominio y utilización de la misma. El hombre parte del cosmos aprenderá a conocerla para adaptarse mejor a él, y para usar en su propio beneficio el dominio, que a través del estudio va adquiriendo de las fuerzas y elementos naturales (...) ejerce una acción tendiente a mejorar nuestras condiciones de vida social y colectiva (SEP, 1935, p. 207)

Si bien Bassols redactó el artículo tercero constitucional socialista, el autor de las ideas centrales de esa propuesta aplicada a la relación sociedad - naturaleza se ha dicho que fue Vicente Lombardo Toledano, ideólogo del socialismo pero realmente fue Enrique Beltrán Castillo, quien es su conferencia de 1935 sobre la educación socialista expuesta en el palacio de Bellas Artes de la Ciudad de México lo señaló así en su conferencia "El lugar de las ciencias biológicas en los programas educativos", él era entonces profesor de la Escuela nacional de maestros.

Toledano era transformista como Engels, esperaba la transformación de la sociedad y su núcleo de interés era la sociedad comunitaria no la relación sociedad medio natural, el medio 
Bio - grafía. Escritos sobre la Biología y su Enseñanza. ISSN 2027-1034

Edición Extraordinaria. p.p. 411 - 419

Memorias del IX Encuentro Nacional de Experiencias en Enseñanza de la Biología y la

Educación Ambiental. IV Congreso Nacional de Investigación en Enseñanza de la Biología.

para él sólo era una fuente de insumos. Beltrán por el contrario era evolucionista y para él era necesario conocer no sólo los nombres o la utilidad económica de las distintas especies, sino el conocimiento de la función dentro de un sistema que cada una tenía incluido el hombre en sociedad. Su mirada es ecologista al cuidar el conjunto a partir de conocer las funciones de cada una de las partes prediciendo que la falta o falla de uno de ellos haría caótico el accionar del todo (ecosistema). Derivado de esa conferencia se cita:

Las ciencias biológicas contribuyen como ninguna otra ciencia, a librarnos de prejuicios religiosos o de otra índole demostrando el carácter unitario de la naturaleza, en la que plantas y animales, incluso el hombre, no son sino manifestaciones distintas en la evolución orgánica a través de las edades incontables. Evolución verificada como resultado de las influencias recíprocas entre el medio y los seres vivientes y estos últimos entre sí, sin ningún fin específico para alcanzar, ni ningún camino especial por recorrer, en respuesta a designios de divinidades absurdas, que no existen más que en función de la ignorancia de los hombres que la inventan. (SEP, 1935, pp. 209-210)

Ese pensamiento era materialista radical como el de su maestro Alfonso L. Herrera quien decía que sólo existía la evolución de la materia, que no había dos tipos de materia viva y no viva sino evolucionada y más evolucionada. En tanto que Lombardo Toledano era transformista como Engels "...en la naturaleza y, por tanto, en la vida social, todo se transforma..." (Lombardo Toledano, 1975, p.19).

Uno de los puntos centrales de ese pensamiento era que la escuela socialista debería de tener un conocimiento concreto de los seres animales y vegetales del país para su aprovechamiento, así como una mentalidad abierta libre de prejuicios para explicar e interpretar el mundo, atacando así la "tendencia funesta al enciclopedismo" (SEP, 1935, p. 232). Esas premisas de la educación socialista desafortunadamente quedaron en plan y no operaron sino a partir de 1939, un solo año escolar, ya que esta tendencia socialista fue derogada a la llegada del nuevo presidente, Caballero de Colón y defensor del catolicismo, Manuel Ávila Camacho (SEP, 1941, 1945)

\section{Ecología y conservacionismo}

Cuevas y Ledesma (2006) proponen que existen cuatro paradigmas fundamentales en la Biología: Evolución, Genética, Teoría celular y Homeostasis, todos ellos entramando una serie de relaciones que dan vida a la ciencia autónoma la Biología. Uno de los aspectos centrales de los paradigmas lo constituye el enfoque homeostático de la vida, si bien Bernard lo propone como una forma de comprender el funcionamiento autorregulado del cuerpo humano, este se puede extender a partir de una visión biologicista a toda la vida, teniendo un uso extendido en Ecología. Ernest Haeckel seguidor de las ideas evolucionistas de Darwin es a quien se le señala como un fundador de la Ecología como ciencia, el estudio del oikos, la casa como un todo ambiental ligado a la vida en su seno pero retroalimentando en todo momento.

La ecología tiene un fondo sistémico, ya que a partir de un pensamiento holístico relaciona las partes de un sistema, las entrama pronosticando que todas están relacionadas entre sí y que al faltar o fallar un elemento del mismo, todo el sistema fallará, los ecosistemas también aluden intercambios energéticos pues es un sistema abierto donde se percibe intercambio de materia y 
Bio - grafía. Escritos sobre la Biología y su Enseñanza. ISSN 2027-1034

Edición Extraordinaria. p.p. 411 - 419

Memorias del IX Encuentro Nacional de Experiencias en Enseñanza de la Biología y la Educación Ambiental. IV Congreso Nacional de Investigación en Enseñanza de la Biología.

energía con el entorno, todo ello depende para su funcionamiento de la sinergia que logre el sistema a partir de la trama de relaciones dinámicas, la energía es esencial y todo sistema tiene su fuente energética regularmente renovable. Todo dentro del sistema debe estar en equilibrio dinámico en tiempo ecológico aludiendo a la homeostasis. Al igual que la teoría de la evolución la ecología era una forma de percibir el medio de forma novedosa.

La racionalidad ilustrada del siglo XVIII implicaba que la naturaleza estaba a disposición del hombre y a partir de su conocimiento y dominio debería de lograrse el progreso. La ilustración movimiento filosófico del siglo XVIII, promovía a la razón como única forma válida de conocer y saber. El conocimiento -racional- daría al hombre en sociedad beneficios, a mayor conocimiento mayor desarrollo, igualando el crecimiento económico con el desarrollo moral, Quesnay proponía que la riqueza de los hombres y del estado está en la naturaleza, como fisiócrata afirmaba que la riqueza de un país reside en la agricultura y el hombre trabajará mejor la tierra que le pertenece no la del Rey (propiedad privada) anteponiéndose a los mercantilistas quienes señalaban el comercio y la industria como valor del Estado.

En Latinoamérica durante la posguerra se promovió a la agricultura extensiva tipo farmer para lograr suficiencia alimentaria, esto provocó el desmonte de zonas boscosas y los monocultivos y en consecuencia un empobrecimiento de la biota, pero también se alentó la industrialización sustitución de importaciones- con lo que centralizó la economía en las ciudades descuidando las zonas rurales y haciendo uso extensivo de los recursos naturales para la elaboración de productos manufacturados en un intento vano de "alcanzar" a las sociedades desarrolladas industrialmente-.

Ese pensamiento o racionalidad ilustrada promovió durante siglos la explotación irracional de los recursos naturales sin percibir o al menos estudiar los efectos que esto tendría en el entorno y el en futuro de los mismos sistemas. El enfoque conservacionista parte de conocer primero lo que se ha de proteger y preservar (es técnico), busca entender nuestro entorno natural al describirlo, explicarlo y predecirlo como un fenómeno en interacción con la sociedad, para formar una cultura de respeto y conservación del medio natural.

\section{Currículo para la formación docente en enseñanza de la Biología}

Durante la gestión del presidente General Manuel Ávila Camacho (1940 - 1946) las instituciones formadoras de docentes para educación primaria se consolidan dando paso a la Escuela Normal Superior (de México), la ley orgánica de la SEP de 1942 le da ese nombre, a pesar de esa nueva denominación, la ENSM ya funcionaba como tal ofreciendo estudios posteriores a la Escuela normal básica para forma maestros de educación secundaria en varias disciplinas. Funcionaba con el plan 1936 de tres años que en 1942 pasa a cuatro y para el caso de las Ciencias biológicas renueva su diseño curricular. La finalidad de esta modificación fue quitar tintes ideológicos y materialistas al currículo de formación de docentes para secundaria, sin embargo por la premura no cubría las necesidades del nuevo sexenio llamado de la unidad. En Marzo de 1945 se da a conocer el nuevo plan de estudios que perduró hasta 1959.Para el caso de ciencias biológicas en la Escuela Normal Superior se organizaba en cuatro bloques pedagógico, disciplinar, afines a la especialidad, cultura general-. En este plan es cuando aparece por primera vez en un currículo en México una asignatura conservacionista, 
Bio - grafía. Escritos sobre la Biología y su Enseñanza. ISSN 2027-1034

Edición Extraordinaria. p.p. 411 - 419

Memorias del IX Encuentro Nacional de Experiencias en Enseñanza de la Biología y la Educación Ambiental. IV Congreso Nacional de Investigación en Enseñanza de la Biología.

"Conservación de recursos naturales" dada su naturaleza tenía un enfoque ecologista (ver Tabla 1).

Lo diseñó e impartió el Dr. Enrique Beltrán Castillo desde su inicio en 1947, hasta 1959 en que el Doctor se incorporó al gabinete del presidente Adolfo López Mateos (1958 - 1964). Para acompañar su curso y dado que no existían textos especializados con el enfoque conservacionista, Beltrán edita tres libros "Los recursos naturales de México y su conservación", guía del curso que se impartía en la Normal superior, además de "La protección a la naturaleza, principios y problemas" y "Los recursos naturales y el futuro de México" como materiales que posteriormente sirvieron de complemento al primero.

\section{Obra e influencia de Beltrán en el conservacionismo}

Enrique Beltrán fue uno de los precursores, a nivel mundial, de la educación ambiental, su formación implicó que su enfoque fuese ecologista - conservacionista, el cual se hace patente en sus obras. En el libro "La protección de la naturaleza" (1949), menciona que la existencia de una gran ciudad significa por eso solo hecho un peligro potencial para los recursos naturales de esa región. En la época desarrollista en México se promueve la creación de grandes ciudades, de ahí ésta preocupación. La filosofía de la conservación con enfoque ecológico es una necesidad para aportar en la solución de los problemas nacionales en este campo, el desconocimiento del fondo ecológico es el problema, la ecología aporta los principios básicos que pueden explicar los problemas de uso y abuso de la naturaleza por variados que sean. Los grandes peligros que el descuido trae acarrean problemas para la protección de los recursos naturales.

Su obra la divide en apartados donde se refleja su intención didáctica. En su temática y en el interior del pequeño texto, se ve una parte descriptiva de las relaciones naturales de la trama vital, de ahí parte para hablar de cada ambiente desde un enfoque conservacionista, señalando el crecimiento de las ciudades y las vías de comunicación como amenazas, ubicándose en el tiempo de la postguerra, en el proyecto de unidad y su ideal desarrollista, oponiendo al saqueo salvaje de los recursos, la idea ecologista del rompimiento de las tramas naturales y de las consecuencia en la misma riqueza de productos naturales de que se dispondrían al futuro.

Tabla 1:

Plan de estudios de la ENS 1945 Maestro en ciencias biológicas. En negritas en el tercer año quinta columna se encuentra la asignatura de Conservación de los recursos naturales de México.

\begin{tabular}{|c|c|c|c|c|}
\hline Año & $\begin{array}{c}\text { Materias } \\
\text { pedagógicas } \\
\text { o generales }\end{array}$ & $\begin{array}{c}\text { Materias de } \\
\text { cultura } \\
\text { general }\end{array}$ & Materias de la especialidad & $\begin{array}{c}\text { Materias } \\
\text { optativa } \\
\text { s afines } \\
\text { a la } \\
\text { especial } \\
\text { idad }\end{array}$ \\
\hline
\end{tabular}


Bio - grafía. Escritos sobre la Biología y su Enseñanza. ISSN 2027-1034

Edición Extraordinaria. p.p. 411 - 419

Memorias del IX Encuentro Nacional de Experiencias en Enseñanza de la Biología y la Educación Ambiental. IV Congreso Nacional de Investigación en Enseñanza de la Biología.

\begin{tabular}{|c|c|c|c|c|c|c|}
\hline 1 & $\begin{array}{l}\text { Conocimiento } \\
\text { de los } \\
\text { adolescentes }\end{array}$ & $\begin{array}{l}\text { Primer curso } \\
\text { de una } \\
\text { lengua } \\
\text { moderna }\end{array}$ & $\begin{array}{l}\text { Primer } \\
\text { curso de } \\
\text { botánica } \\
\text { (botánica } \\
\text { general) }\end{array}$ & $\begin{array}{l}\text { Primer } \\
\text { curso de } \\
\text { Zoología } \\
\text { (zoología } \\
\text { general) }\end{array}$ & $\begin{array}{l}\text { Anatomía } \\
\text { fisiología e } \\
\text { higiene }\end{array}$ & $\begin{array}{l}\text { Optativa } \\
\text { I }\end{array}$ \\
\hline 2 & $\begin{array}{l}\text { Educación de } \\
\text { los } \\
\text { adolescentes }\end{array}$ & $\begin{array}{l}\text { Segundo } \\
\text { curso de una } \\
\text { lengua } \\
\text { moderna }\end{array}$ & $\begin{array}{l}\text { Segundo } \\
\text { curso de } \\
\text { botánica } \\
\text { (criptógama } \\
\text { s) }\end{array}$ & $\begin{array}{l}\text { Segundo } \\
\text { curso de } \\
\text { Zoología } \\
\text { (Zoología } \\
\text { de } \\
\text { invertebra } \\
\text { dos) }\end{array}$ & $\begin{array}{l}\text { Higiene y } \\
\text { primeros } \\
\text { auxilios }\end{array}$ & $\begin{array}{l}\text { Optativa } \\
\text { II }\end{array}$ \\
\hline \multirow[t]{2}{*}{3} & $\begin{array}{l}\text { Didáctica } \\
\text { general }\end{array}$ & \multirow[t]{2}{*}{$\begin{array}{l}\text { Teoría del } \\
\text { conocimiento } \\
\text { y } \\
\text { metodología } \\
\text { de las } \\
\text { ciencias }\end{array}$} & \multirow[t]{2}{*}{$\begin{array}{l}\text { Tercer } \\
\text { curso de } \\
\text { botánica } \\
\text { (fanerógam } \\
\text { as) }\end{array}$} & \multirow[t]{2}{*}{$\begin{array}{l}\text { Tercer } \\
\text { curso de } \\
\text { zoología } \\
\text { (Vertebrad } \\
\text { os) }\end{array}$} & $\begin{array}{l}\text { Conservació } \\
\text { n de los } \\
\text { recursos } \\
\text { naturales de } \\
\text { México }\end{array}$ & \\
\hline & $\begin{array}{l}\text { Didáctica de la } \\
\text { materia de la } \\
\text { especialidad }\end{array}$ & & & & $\begin{array}{l}\text { Historia de } \\
\text { las ciencias } \\
\text { biológicas }\end{array}$ & \\
\hline \multirow[t]{2}{*}{4} & $\begin{array}{l}\text { Orientación } \\
\text { pedagógica } \\
\text { profesional y } \\
\text { psicotécnica }\end{array}$ & $\begin{array}{l}\text { Curso } \\
\text { superior de } \\
\text { español }\end{array}$ & \multirow[t]{2}{*}{$\begin{array}{l}\text { Técnicas de } \\
\text { laboratorio }\end{array}$} & \multirow[t]{2}{*}{$\begin{array}{l}\text { Biología } \\
\text { general }\end{array}$} & \multirow[t]{2}{*}{$\begin{array}{l}\text { Paleontologí } \\
\text { a }\end{array}$} & \\
\hline & $\begin{array}{l}\text { Historia de los } \\
\text { sistemas } \\
\text { educativos de } \\
\text { la segunda } \\
\text { enseñanza }\end{array}$ & Etimologías & & & & \\
\hline
\end{tabular}

Por ello señalaba que:

[...] pero sabemos igualmente que si movidos por torpes apetitos de momentos, orientamos, nuestras explotaciones en forma desmesurada y destructiva, podemos adquirir la riqueza momentánea del salteador que se apropia violentamente el rico botín producto del despojo, pero estaremos abriendo también la puerta de la miseria, que se perfilaría en el horizonte y que, si a veces no llega a alcanzarnos a nosotros mismos, para imponernos el castigo a que nos hicimos acreedores, en cambio será compañera inseparable de nuestros hijos, que maldecirán la codicia, y aún a veces la simple ignorancia, de quienes no pudieron comprender que no eran los propietarios absolutos 
Bio - grafía. Escritos sobre la Biología y su Enseñanza. ISSN 2027-1034

Edición Extraordinaria. p.p. 411 - 419

Memorias del IX Encuentro Nacional de Experiencias en Enseñanza de la Biología y la Educación Ambiental. IV Congreso Nacional de Investigación en Enseñanza de la Biología.

de las riquezas naturales que detentaban, sino simplemente sus custodios, que habiéndolas recibido de sus antepasados, estaban obligados a conservarlos intactos, o a aumentarlos si era posible, para legarlas a sus descendientes (Beltrán, 1949, pp. 89-90).

La educación conservacionista embrión de la Educación Ambiental que había generado en México el Dr. Beltrán, se impulsó en los planes de estudio de la ENSM en la carrera de Ciencias biológicas y posteriormente en Biología, a su salida de la escuela, para ocupar cargos de responsabilidad federal en el gabinete del presidente Adolfo López Mateos (1958 - 1964) sus predecesores, alumnos formados bajo su sombra prosiguieron con su obra, impartiendo esa materia hasta la modificación curricular de los 70 (Beltrán, 1977). Dada la relevancia de su aporte pro conservacionista a Beltrán se le otorgó "Premio al mérito en la enseñanza de la conservación" por parte de la Unión panamericana y otro por la National Association of Biology Teachers. Además era representante del área de ciencias biológicas en el consejo de la Escuela Normal Superior de México.

\section{CONCLUSIONES}

La propuesta educativa socialista buscaba que la educación aportara en el conocimiento uso y explotación de los recursos naturales de nuestro país, una racionalidad ilustrada favorecida por el desarrollismo, la contraparte que desde la educación se da a esa anunciada barbarie ecocida la ofrece Enrique Beltrán con esta propuesta conservacionista, conocer y explotar sí, pero racionalmente. Sin embargo los planes educativos incrementaron la visión utilitaria de la naturaleza anteponiendo el criterio económico al naturalista, bien fomentando monocultivos o explotando especies silvestres sin la racionalidad ecologista y respondiendo a las demandas alimentarias y económicas inmediatas de la sociedad. El aporte de Beltrán novedoso en los 40 no tuvo eco en la política educativa ni económica del país pero sentó las bases de una educación en pro del medio ambiente. 
Bio - grafía. Escritos sobre la Biología y su Enseñanza. ISSN 2027-1034

Edición Extraordinaria. p.p. 411 - 419

Memorias del IX Encuentro Nacional de Experiencias en Enseñanza de la Biología y la

Educación Ambiental. IV Congreso Nacional de Investigación en Enseñanza de la Biología.

\section{REFERENCIAS}

Beltrán, E. (1949). La protección de la naturaleza. Principios y problemas. Biblioteca enciclopédica popular. México: SEP.

Beltrán, E. (1977). Medio siglo de recuerdos de un biólogo mexicano. México: Sociedad Mexicana de Historia Natural.

Cuevas, C. y Ledesma, I. (2006). Alfonso L. Herrera, controversias y debates durante el inicio de la Biología en México. Historia mexicana, vol L, n ${ }^{\circ}$, enero - marzo pp 973 - 1013. México: El colegio de México.

Lombardo - Toledano, V. (1975). La política y las leyes de la realidad”, en ¿Moscú o Pekín? La vía mexicana al socialismo. México: Editorial Combatiente.

SEP (1935). Memoria relativa al estado que guarda el ramo de educación pública. El 31 de agosto de 1935. Tomo II. Documentos SEP. México: SEP.

SEP (1937). Memorias de la Secretaría de Educación Pública, de septiembre de 1936 a agosto de 1937. Tomo II. México: SEP.

SEP (1941). La educación pública en México, desde el $1^{\circ}$ de diciembre de 1934 hasta el 30 de noviembre de 1940. Tomo II. México: SEP.

SEP (1945). Segundo congreso nacional de educación normal. Tomo II. México: SEP.

SEP (1948). Memoria de la Secretaría de Educación Pública, 1947 - 1948. México: SEP. 